\title{
Oral glucose tolerance test (OGTT)
}

National Diabetes Information Clearinghouse (NDIC)

\section{Definitions}

Pre-diabetes

Defined by National Diabetes Information Clearinghouse (NDIC)

Diabetes

Defined by National Diabetes Information Clearinghouse (NDIC)

\section{Glucose}

Defined by National Diabetes Information Clearinghouse (NDIC)

\section{Source}

National Diabetes Information Clearinghouse (U.S.). (2009). The diabetes dictionary. [Bethesda, Md.]: U.S. Dept. of Health and Human Services, National Institutes of Health, National Institute of Diabetes and Digestive and Kidney Diseases, National Diabetes Information Clearinghouse.

A test to diagnose pre-diabetes and diabetes. The oral glucose tolerance test is given by a health care professional after an overnight fast. A blood sample is taken and then the patient drinks a high-glucose beverage. Blood samples are taken at hourly intervals for 2 to 3 hours. Test results are compared with a standard and show how the body uses glucose over time. 